\title{
Ciclo térmico para la obtención de una ferrita de Ni-Zn: (II) Influencia de la etapa de enfriamiento
}

\author{
A. BARBA, C. CLAUSELL, M. MONZÓ, J.C JARQUE \\ Instituto de Tecnología Cerámica. Universitat Jaume I. Castellón. Spain.
}

\begin{abstract}
Las piezas cerámicas obtenidas a partir de polvos de ferritas de composición $\left(\mathrm{Cu}_{0.12} \mathrm{Ni}_{0.23} \mathrm{Zn}_{0.65}\right) \mathrm{Fe}_{2} \mathrm{O}_{4}$ destacan como absorbentes de radiación electromagnética entre las ferritas de $\mathrm{Ni}$ - $\mathrm{Zn}$ dopadas con $\mathrm{Cu}$, impidiendo interferencias entre dispositivos electrónicos. En un trabajo previo se estableció la metodología para diseñar la etapa de sinterización de un ciclo térmico que permite obtener las piezas cerámicas mencionadas con una microestructura adecuada (elevada densidad relativa y escaso crecimiento de grano) para que presenten unas buenas propiedades electromagnéticas. En este trabajo se ha estudiado la etapa de enfriamiento del mismo ciclo térmico (etapa siguiente a la de sinterización), determinando la influencia que ejerce la velocidad de enfriamiento sobre la densidad relativa en cocido, el tamaño medio de grano y la distribución de tamaños de grano, variando el tiempo de sinterización del ciclo térmico empleado
\end{abstract}

Palabras clave: ferritas, ciclo térmico, velocidad de enfriamiento.

Thermal cycle for obtaining a Ni-Zn ferrite: (II) Influence of the cooling stage.

Ceramic bodies formed from $\left(\mathrm{Cu}_{0.12} \mathrm{Ni}_{0.23} \mathrm{Zn}_{0.65}\right) \mathrm{Fe}_{2} \mathrm{O}_{4}$ ferrite powders stand out as electromagnetic radiation absorbers among $\mathrm{Cu}$-doped $\mathrm{Ni}-\mathrm{Zn}$ ferrites, preventing interference between electronic devices. In a preliminary study, a methodology was established for designing the sintering stage of a thermal cycle, which allows the foregoing ceramic bodies to be obtained with the appropriate microstructure (high relative density and little grain growth) to provide them with good electromagnetic properties. The present study examines the cooling stage (i.e. the stage following the sintering stage) of that thermal cycle and the influence of the cooling rate on fired relative density, average grain size, and grain size distribution, varying the sintering time of the thermal cycle used.

Keywords: ferrites, thermal cycle, cooling rate.

\section{INTRODUCCIÓN}

Los polvos cerámicos conocidos genéricamente como ferritas son, básicamente, óxidos mixtos de hierro y de uno o más elementos metálicos. Las ferritas de Ni-Zn, con diferentes porcentajes de $\mathrm{Cu}$, suelen utilizarse para la fabricación de piezas cuya función es la de impedir interferencias entre los dispositivos eléctricos o electrónicos, dada su capacidad de absorción de ondas electromagnéticas (1-3). Las propiedades magnéticas de estas piezas quedan determinadas tanto por su composición química como por su microestructura (4-7).

Las piezas de ferrita se fabrican a escala industrial mediante el denominado proceso cerámico tradicional $(8,9)$. Con el tratamiento térmico se debe: $\mathrm{i} /$ conseguir que las piezas alcancen la máxima densidad relativa posible; ii/ aumentar el tamaño de los granos de forma homogénea, manteniendo un tamaño de grano relativamente pequeño y una distribución de tamaños de grano estrecha; iii / evitar un crecimiento de grano exagerado. La temperatura máxima de sinterización y el tiempo de permanencia a dicha temperatura son las dos variables más relevantes para controlar la sinterización de este tipo de ferritas $(6,7)$.

Los autores han estudiado la síntesis (10), la sinterización (11-14) y las propiedades (15) de ferritas de Ni-Zn dopadas con $\mathrm{Cu}$. En un trabajo previo (16), a partir de las velocidades de densificación y de crecimiento de grano, se ha diseñado la etapa de sinterización de un ciclo térmico para la obtención de piezas de ferrita de composición aproximada $\left(\mathrm{Cu}_{0.12} \mathrm{Ni}_{0.23} \mathrm{Zn}_{0.65}\right)$ $\mathrm{Fe}_{2} \mathrm{O}_{4}$. Así, se ha encontrado la variación que la temperatura de sinterización debe seguir a medida que avanza el tiempo de sinterización para que, en cada instante, se mantenga una velocidad de densificación relativamente alta evitando, simultáneamente, que una elevada velocidad de crecimiento de grano provoque un crecimiento irregular y exagerado de los mismos.

En este trabajo se ha estudiado el efecto que el número de tramos a temperatura constante, que constituyen la etapa de sinterización, y la velocidad de enfriamiento tienen sobre la 
densidad relativa en cocido, el tamaño medio de grano y la amplitud de la distribución de tamaños de grano en una ferrita de igual composición a la empleada en el trabajo previo (16).

\section{PROCEDIMIENTO Y RESULTADOS EXPERIMENTA- LES}

Como material de partida se ha utilizado un polvo atomizado industrial de ferrita, obtenido previamente por síntesis a partir los óxidos correspondientes, de composición $\left(\mathrm{Cu}_{0.12} \mathrm{Ni}_{0.23} \mathrm{Zn}_{0.65}\right) \mathrm{Fe}_{2} \mathrm{O}_{4}$. Con éste se han conformado piezas cilíndricas de $2 \mathrm{~cm}$ de diámetro y $3 \mathrm{~mm}$ de espesor, por prensado en seco unidireccional a una presión de $2000 \mathrm{~kg} / \mathrm{cm}^{2}$ para conseguir una densidad relativa en seco del 0.61 (16). Las muestras se sinterizaron en un horno eléctrico de laboratorio, en atmósfera de aire, utilizando ocho ciclos térmicos diferentes de tres etapas:

i/ una etapa de calentamiento, idéntica para todos los ciclos térmicos, consistente en un calentamiento desde temperatura ambiente hasta $500^{\circ} \mathrm{C}$, a una velocidad de $12^{\circ} \mathrm{C} / \mathrm{min}$, un tiempo de permanencia a dicha temperatura de 2 horas (para oxidar los aditivos orgánicos utilizados para obtener el polvo atomizado y facilitar la operación de prensado) y un segundo calentamiento desde $500^{\circ} \mathrm{C}$ hasta $1100^{\circ} \mathrm{C}$; también a la velocidad de $12^{\circ} \mathrm{C} / \mathrm{min}$. En consecuencia, en los ocho ciclos térmicos ensayados el tiempo de la etapa de calentamiento fue 3.5 horas.

ii / una etapa de sinterización, con uno o varios tramos de temperatura constante (cada tramo a una temperatura diferente que se denomina $\mathrm{T}_{\max }$ ) y un tiempo de permanencia a cada una de esas temperaturas $\left(t_{T}\right)$, siendo el tiempo de sinterización $\left(\mathrm{t}_{\text {sinterización }}\right)$ la suma de todos los $\mathrm{t}_{\mathrm{T}}$ según:

$$
\mathrm{t}_{\text {sinterización }}=\sum \mathrm{t}_{\mathrm{T}}(\mathrm{h})
$$

iii/ una etapa de enfriamiento, a una velocidad determinada (v), desde el tramo de la etapa de sinterización de menor temperatura $\left[\left(\mathrm{T}_{\max }\right)_{\min }\right]$ hasta los $800^{\circ} \mathrm{C}$ (en el caso de velocidades de enfriamiento grandes) o hasta los $250^{\circ} \mathrm{C}$ (en el caso de velocidades de enfriamiento pequeñas), continuando con un enfriamiento natural hasta alcanzar la temperatura ambiente. El tiempo de la etapa de enfriamiento se ha determinado experimentalmente en cada uno de los casos.

El tiempo del ciclo térmico se obtiene como la suma de los tres tiempos mencionados anteriormente:

$$
\begin{aligned}
& \mathrm{t}_{\text {ciclo }}=\mathrm{t}_{\text {calentamiento }}+\mathrm{t}_{\text {sinterización }}+\mathrm{t}_{\text {enfriamiento }}= \\
& \mathrm{t}_{\text {calentamie nto }}+\sum \mathrm{t}_{\mathrm{T}}+\mathrm{t}_{\text {enfriamien to }}
\end{aligned}
$$

De acuerdo con los resultados obtenidos en un trabajo previo (16) se han ensayado etapas de sinterización constituidas por hasta cuatro tramos de temperatura constante, a las temperaturas $\left(\mathrm{T}_{\max }\right)$ de $1100,1080,1060$ y $1040^{\circ} \mathrm{C}$. Asimismo, se han ensayado dos velocidades de enfriamiento: 2 y $20^{\circ} \mathrm{C} / \mathrm{min}$. En la Tabla I se muestran los ocho ciclos térmicos ensayados. En la primera columna se detalla la referencia del ciclo: el primer dígito indica el número de tramos a temperatura constante que constituyen la etapa de sinterización y el segundo dígito señala la velocidad de enfriamiento empleada tras la etapa de sinterización. En las columnas segunda y tercera se detalla, para cada ciclo térmico, las temperaturas $\left(\mathrm{T}_{\max }\right)$ y el tiempo de permanencia $\left(t_{T}\right)$ a cada temperatura de cada uno de los tramos de temperatura constante que constituyen la etapa de sinterización. En las columnas cuarta, quinta, sexta y séptima se muestran, respectivamente, el tiempo de sinterización (calculado mediante la ecuación [1]), el tiempo de calentamiento (3.5 horas en todos los casos), el tiempo de enfriamiento (medido experimentalmente) y el tiempo del ciclo térmico (calculado mediante la ecuación [2]).

En cada ciclo térmico se han tratado cuatro piezas. De cada pieza sinterizada se ha determinado la densidad aparente $\left(\rho_{\mathrm{ap}}\right)$, la densidad relativa $\left(\phi_{c}\right)$, el tamaño medio de grano $\left(G_{50}\right)$ y la amplitud de la distribución de tamaños de grano $\left(\mathrm{S}_{\mathrm{G}}\right)$, siguiendo los procedimientos utilizados en trabajos anteriores $(12,15$, 16). En la Tabla I (columna octava, novena y décima) se muestran los valores medios de los resultados obtenidos para estos tres parámetros.

TABLA I. CICLOS TÉRMICOS ENSAYADOS Y VALORES EXPERIMENTALES DE LA DEN-

\begin{tabular}{|c|c|c|c|c|c|c|c|c|c|}
\hline Ciclo & $\begin{array}{l}\mathrm{T}_{\max } \\
\left({ }^{\circ} \mathrm{C}\right)\end{array}$ & $\begin{array}{l}t_{T} \\
\text { (h) }\end{array}$ & $\begin{array}{c}t_{\text {sinterizacion }} \\
t_{T}(h)\end{array}$ & $\begin{array}{l}\mathrm{t}_{\text {calenta- }} \\
\text { miento } \\
\text { (h) }\end{array}$ & $\begin{array}{l}t_{\text {enfria- }} \\
\text { miento } \\
\text { (h) }\end{array}$ & $\begin{array}{l}\mathrm{t}_{\text {cidlo }} \\
\text { (h) }\end{array}$ & $\phi_{c}$ & $\begin{array}{c}\mathrm{G}_{50} \\
(\mu \mathrm{m})\end{array}$ & $\begin{array}{c}S_{G} \\
(\mu \mathrm{m})\end{array}$ \\
\hline $1(2)$ & 1100 & 0.1 & 0.1 & 3.5 & 8.0 & 11.6 & 0.937 & 5.6 & 7.1 \\
\hline \multirow{2}{*}{$2(2)$} & 1100 & 0.1 & \multirow{2}{*}{0.4} & \multirow{2}{*}{3.5} & \multirow{2}{*}{8.0} & \multirow{2}{*}{11.9} & \multirow{2}{*}{0.938} & \multirow{2}{*}{7.5} & \multirow{2}{*}{9.0} \\
\hline & 1080 & 0.3 & & & & & & & \\
\hline \multirow{3}{*}{$3(2)$} & 1100 & 0.1 & \multirow{3}{*}{2.1} & \multirow{3}{*}{3.5} & \multirow{3}{*}{8.0} & \multirow{3}{*}{13.6} & \multirow{3}{*}{0.944} & \multirow{3}{*}{8.7} & \multirow{3}{*}{12.0} \\
\hline & 1080 & 0.3 & & & & & & & \\
\hline & 1060 & 1.7 & & & & & & & \\
\hline \multirow{4}{*}{$4(2)$} & 1100 & 0.1 & \multirow{4}{*}{7.9} & \multirow{4}{*}{3.5} & \multirow{4}{*}{8.0} & \multirow{4}{*}{19.4} & \multirow{4}{*}{0.955} & \multirow{4}{*}{10.9} & \multirow{4}{*}{14.0} \\
\hline & 1080 & 0.3 & & & & & & & \\
\hline & 1060 & 1.7 & & & & & & & \\
\hline & 1040 & 5.8 & & & & & & & \\
\hline $\begin{array}{c}1 \\
(20)\end{array}$ & 1100 & 0.1 & 0.1 & 3.5 & 4.6 & 8.2 & 0.916 & 4.4 & 5.4 \\
\hline \multirow{2}{*}{$\begin{array}{c}2 \\
(20)\end{array}$} & 1100 & 0.1 & \multirow{2}{*}{0.4} & \multirow{2}{*}{3.5} & \multirow{2}{*}{4.6} & \multirow{2}{*}{8.5} & \multirow{2}{*}{0.930} & \multirow{2}{*}{6.2} & \multirow{2}{*}{6.6} \\
\hline & 1080 & 0.3 & & & & & & & \\
\hline \multirow{3}{*}{$\begin{array}{c}3 \\
(20)\end{array}$} & 1100 & 0.1 & \multirow{3}{*}{2.1} & & & & & & \\
\hline & 1080 & 0.3 & & 3.5 & 4.6 & 10.2 & 0.944 & 9.1 & 9.9 \\
\hline & 1060 & 1.7 & & & & & & & \\
\hline & 1100 & 0.1 & & & & & & & \\
\hline 4 & 1080 & 0.3 & 79 & 35 & 46 & 160 & 0951 & 113 & 128 \\
\hline (20) & 1060 & 1.7 & 1.9 & 0.0 & 4.0 & 10.0 & 0.951 & 11.3 & 12.0 \\
\hline & 1040 & 5.8 & & & & & & & \\
\hline
\end{tabular}
SIDAD RELATIVA EN COCIDO, EL TAMAÑO MEDIO DE GRANO Y LA AMPLITUD DE LA DISTRIBUCIÓN DE TAMAÑOS DE GRANO ALCANZADOS EN CADA UNO.

\section{ANÁLISIS DE LOS RESULTADOS}

La Figura 1 muestra la evolución de la densidad relativa en cocido en función del tiempo de sinterización (columnas 8 y 4 de la Tabla I) para las dos velocidades ensayadas en la etapa de enfriamiento. Como puede observarse, la densidad relativa en cocido aumenta con el tiempo de sinterización, tendiendo hacia un valor constante. A tiempos de sinterización bajos, la densidad relativa de las piezas cocidas aumenta con la disminución de la velocidad de la etapa de enfriamiento, tendiendo a igualarse los valores de la densidad relativa a medida que aumenta el tiempo de sinterización. 


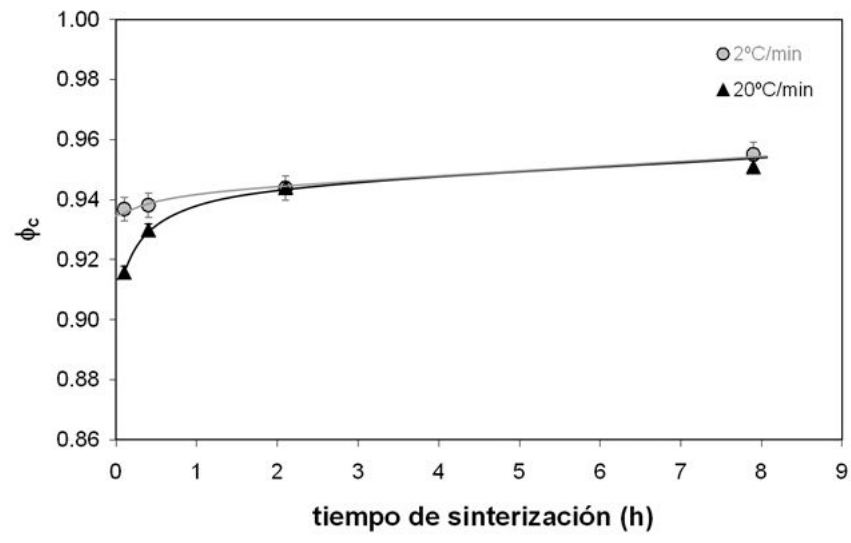

Fig. 1- Evolución de la densidad relativa en cocido $\left(\phi_{c}\right)$ con el tiempo de sinterización para los ciclos térmicos ensayados.

La Figura 2 muestra la evolución del tamaño medio de grano y de la amplitud de la distribución de tamaños de grano en función del tiempo de sinterización (columnas 9, 10 y 4 de la Tabla I) para las dos velocidades de enfriamiento ensayadas. Del análisis de esta gráfica se desprende que tanto el tamaño medio de grano como la amplitud de la distribución de los tamaños de grano, aumentan con el tiempo de sinterización, tendiendo, en ambos casos, hacia un valor constante. Asimismo, se observa que, en el caso de tiempos de sinterización bajos, tanto el tamaño medio de grano como la amplitud de la distribución de tamaños de grano disminuyen al aumentar la velocidad de enfriamiento utilizada. En el caso de tiempos de sinterización altos, la amplitud de tamaños de grano disminuye con el aumento de la velocidad de enfriamiento, mientras que el tamaño medio de grano permanece constante.

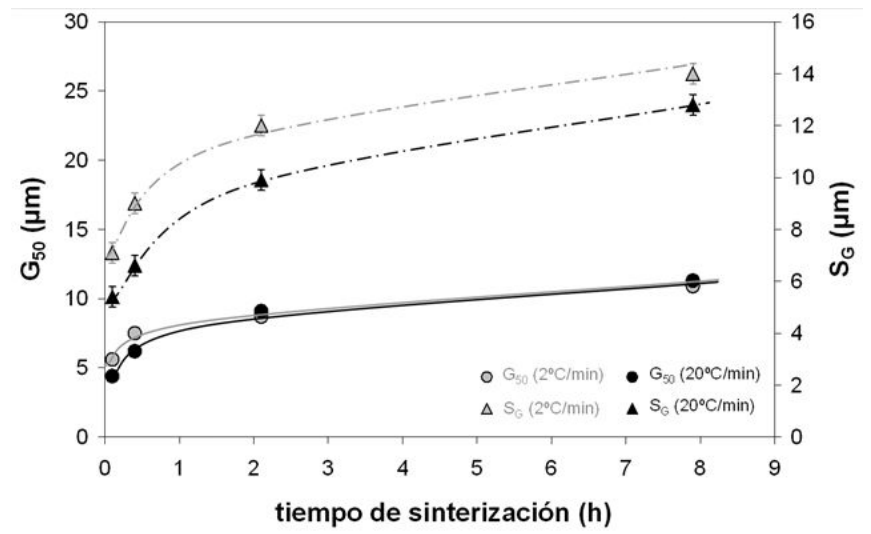

Fig. 2- Evolución del tamaño medio de grano $\left(G_{50}\right)$ con el tiempo de sinterización para los ciclos térmicos ensayados.

En la Figura 3 se muestra la evolución del tamaño medio de grano con la densidad relativa en cocido, para las dos velocidades de enfriamiento ensayadas, observándose como el tamaño medio de grano aumenta con el incremento de la densidad relativa en cocido, y disminuye ligeramente con el incremento de la velocidad de la etapa de enfriamiento.

En la Figura 4 se recogen las imágenes de las microestructuras de las ocho muestras obtenidas. En dichas imágenes se aprecia como la distribución de tamaños de grano se hace más fina y más estrecha al reducir el número de tramos a temperatura constante (reducción del tiempo de sinterización). Al comparar las dos velocidades de enfriamiento se observa la misma

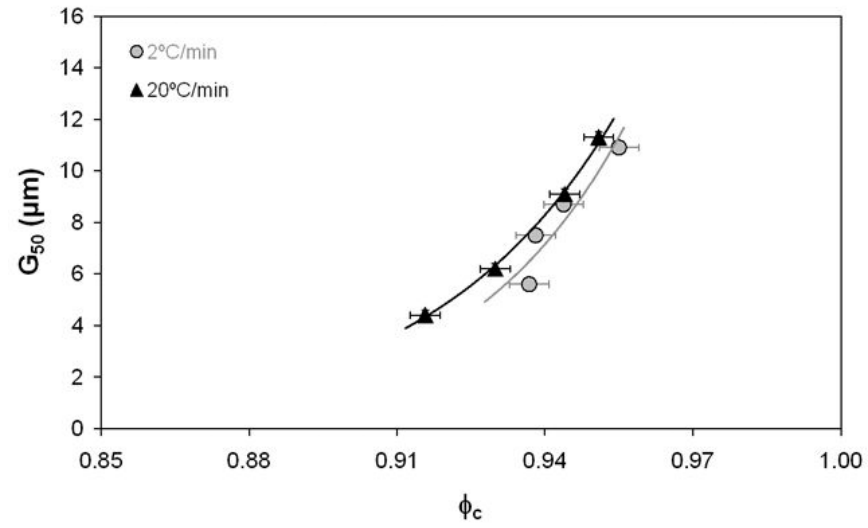

Fig. 3- Evolución del tamaño medio de grano $\left(G_{50}\right)$ con la densidad relativa en cocido $\left(\phi_{c}\right)$ para los ciclos térmicos ensayados.

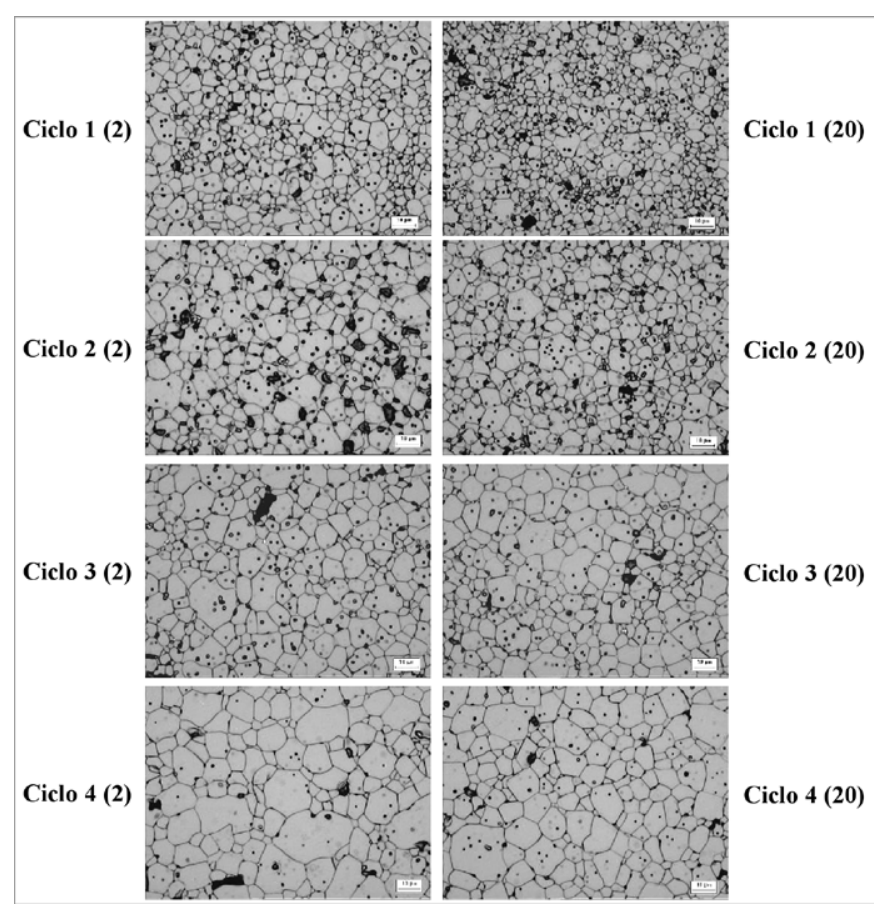

Fig. 4- Microestructura en cocido de las piezas sinterizadas para los ciclos térmicos ensayados.

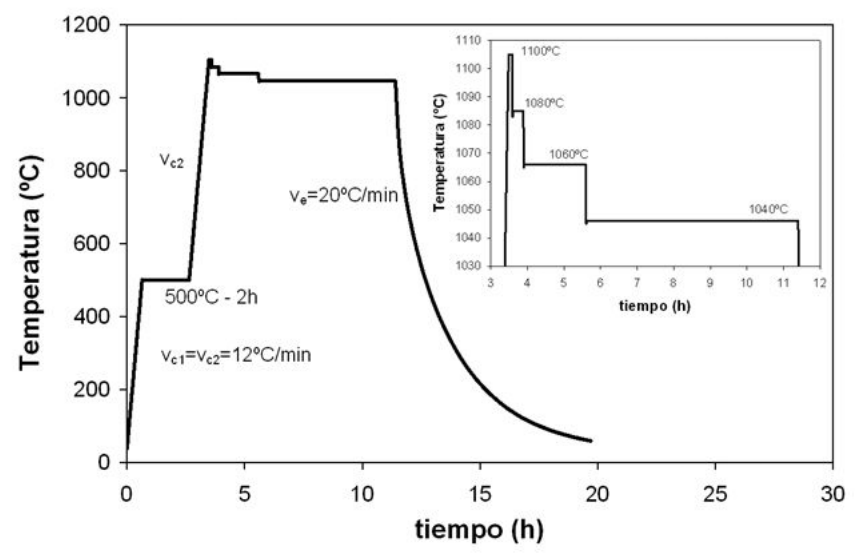

Fig. 5- Ciclo térmico propuesto. 
tendencia pero de forma menos acusada. Estas observaciones cualitativas corroboran los resultados cuantitativos mostrados en las Figuras 1 y 2 y descritos en los párrafos anteriores.

A la vista de los resultados obtenidos se puede proponer, para la sinterización de piezas de ferrita de composición y microestructura en crudo similar a las del presente trabajo, un ciclo térmico como el mostrado en la Figura 5, con cuatro tramos a temperatura constante (entre $1100^{\circ} \mathrm{C}$ y $1040^{\circ} \mathrm{C}$ ), con un tiempo de sinterización de aproximadamente 8 horas, una velocidad de enfriamiento de $20^{\circ} \mathrm{C} / \mathrm{min}$ y un tiempo total del ciclo térmico de 16 horas. Esta elevada velocidad de enfriamiento permite no solo disminuir el tiempo de ciclo térmico sino mantener una microestructura adecuada en cuanto al tamaño medio de grano se refiere, e incluso mejorarla al obtener una amplitud de la distribución de tamaños de grano más estrecha.

\section{CONCLUSIONES}

Para la ferrita de composición $\left(\mathrm{Cu}_{0.12} \mathrm{Ni}_{0.23} \mathrm{Zn}_{0.65}\right) \mathrm{Fe}_{2} \mathrm{O}_{4^{\prime}}$ y en las condiciones de operación estudiadas:

1. Se ha completado el estudio realizado en un trabajo previo (16), adecuando la velocidad de enfriamiento (dentro de los límites posibles) para obtener la mejor microestructura (similar a la de la ferrita industrial), es decir, elevada densidad relativa y escaso crecimiento de grano, de acuerdo también con la experiencia previa del grupo (15).

2. Se ha comprobado la conveniencia de utilizar velocidades de enfriamiento elevadas dado que permiten disminuir tanto el tiempo del ciclo térmico como la amplitud de la distribución de tamaños de grano.

\section{BIBLIOGRAFÍA}

1. K.Y. Kim, W.S. Kim, Y.D. Ju, H.J. Jung, Effect of addition of the Cuo- $\mathrm{Fe}_{2} \mathrm{O}_{3}$ system on the electromagnetic wave absorbing properties of sintered ferrite, J. Mater. Sci., 27, 4741-4745 (1992)

2. W.S. Kim, S.J. Yoon, K.Y. Kim, Effects of sintering conditions of sintered Ni$\mathrm{Zn}$ ferrites on properties of electromagnetic wave absorber, Mater. Lett., 19, 3-4, 149-155 (1994)

3. S.R. Murthy, Low temperature sintering of NiCuZn ferrite and its electrical, magnetic and elastic properties, J. Mater. Sci. Lett., 21, 8, 657-660 (2002)

4. H. Igarashi, K. Okazaki, Effects of porosity and grain size on the magnetic properties of NiZn ferrite, J. Am. Ceram. Soc., 60, 1-2, 51-54 (1977)

5. SH. Bashkirov, A.B. Liberman, A.M. Khasanov, Effect of sintering temperature on crystalline and magnetic structure of nickel zinc ferrites, Soviet Powder Metallurgy and Metal Ceramics (EnglishTranslation), 27, 5, 367-370 (1988)

6. J. Sanyal. Manufacture of ferrites, pp. 496-505 in S. KUMAR. (ed. lit.) Handbook of ceramics: section B. Kumar \& associates, Calcutta, 1995

7. J. Kulikowski, A. Lesniewski, Properties of Ni-Zn ferrites for magnetic heads: technical possibilities and limitations, J. Magn. Magn. Mater., 19, 117-119 (1980)

8. J.L. Hervás, J. Albors, La industria cerámica europea en el siglo XXI: Retos tecnológicos y desafíos de la próxima década, Bol. Soc. Esp. Ceram. V., 45, $1,13-21(2006)$

9. A. Barba, From chemical engineering to ceramic technology: a review of research at the Instituto de Tecnología Cerámica, Bol. Soc. Esp. Ceram. V., 44, 3, 155-168 (2005)

10. A. Barba, M.J. Orts, E. Sánchez, C. Clausell. Kinetic model applicable to synthesis of $\left(\mathrm{Cu}_{0.25} \mathrm{Ni}_{0.25} \mathrm{Zn}_{0.50}\right) \mathrm{Fe}_{2} \mathrm{O}_{4}$ ferrite. Br. Ceram. Trans., 99(2), 53-56 (2000).

11. A. Barba, C. Clausell, C. Felíu, M. Monzó. Influencia del tamaño de partícula, de la microestructura en crudo y del tratamiento térmico sobre la microestructura en cocido de una ferrita de Ni-Zn, pp. 317-324 en VIII Congreso Nacional de Materiales: Universidad Politécnica de Valencia, Valencia, 2004

12. A. Barba, C. Clausell, C. Felíu, M. Monzó, Sintering of $\left(\mathrm{Cu}_{0.25} \mathrm{Ni}_{0.25} \mathrm{Zn}_{0.50}\right)$ $\mathrm{Fe}_{2} \mathrm{O}_{4}$ Ferrite, J. Am. Ceram. Soc., 87, 4, 571-577 (2004)

13. A. Barba, C. Clausell, M. Monzó, V. Cantavella. Study of the sintering of ferrites of composition $\left(\mathrm{Cu}_{0.25} \mathrm{Ni}_{0.25} \mathrm{Zn}_{0.50}\right) \mathrm{Fe}_{2} \mathrm{O}_{4^{\prime}}$ pp. 185 en X Congreso Mediterráneo de Ingeniería Química: Ingeniería química y vida: abstracts: Fira de Barcelona, Barcelona, 2005

14. A. Barba, C. Clausell, M. Monzó, V. Cantavella. Diseño del ciclo térmico de una ferrita, de composición $\left(\mathrm{Cu}_{025} \mathrm{Ni}_{025} \mathrm{Zn}_{050}\right) \mathrm{Fe}_{2} \mathrm{O}_{4}$, basado en su cinética de sinterización, en A. Ramírez de Arellano et. al. (Eds.) Innovación, ciencia y tecnología de los materiales cerámicos y vítreos 2005: Libro de resúmenes de los trabajos presentados a la XLV reunión anual de la Sociedad Española de Cerámica y Vidrio: SECV, Sevilla, 2005

15. A. Barba, C. Clausell, C. Felíu, M. Monzó, L. Nuño, D. Heras, J.V. Balbastre, Study of NiZn Ferrite Complex Permeability: Effect of relative density and microstructure, J. Am. Ceram. Soc., 87, 7, 1314-1318 (2004)

16. A. Barba, C. Clausell, M. Monzó, J.C. Jarque, Ciclo térmico para la obtención de una ferrita de Ni-Zn: (I) Diseño de la etapa de sinterización, Bol. Soc. Esp. Ceram. V., 47, 1, 13-23 (2008)

Recibido: 19.10 .07

Aceptado: 04.03 .08 